\title{
Análisis de estructuras algebraicas mediante la modelización de puzzles y rompecabezas*
}

\author{
María José Felipe $^{1}$ y Víctor Manuel Ortiz Sotomayor ${ }^{2}$ \\ ${ }^{1}$ Depto. Matemática Aplicada, ETS Ingeniería Industrial, Universitat Politècnica de \\ València \\ ${ }^{2}$ Instituto Universitario de Matemática Pura y Aplicada, Universitat Politècnica de \\ València
}

\begin{abstract}
The subject Algebraic Methods and their Applications of the Master in Mathematical Research is focused on showing the applicability that some abstract algebraic structures have in various scientific areas, in both pure mathematics and other more applied contexts of real life. One of the main troubles when learning these structures is their high degree of abstraction which difficults the understanding, specially for beginner students in this topic, as well as the lack of applicability they have been treated before, focusing only in the mathematical aspect.

The method of using games in teaching is an innovative and effective tool known as "game-based learning". This pedagogic tendency is used as a playful component in a concrete learning objective. In this direction, the present paper concerns about the mathematical modelling of different puzzles and brainteasers, as well as the analysis of their solubility, through algebraic techniques that have been used in the aforementioned subject. The goal is to address the study of both the structure of a group and also its most basic properties. To do this, we make use in the classroom of the computational algebraic system GAP (Groups, Algorithms and Programming), which eases the computations in discrete algebra and which helps in a better understunding of this abstract structure. The case of the Rubik's cube, one of the most celebrated and best-selling games in the world, and its variations are specially considered.
\end{abstract}

Keywords: Computational algebra, group theory, modelling, puzzles, GAP.

\footnotetext{
* El primer autor quiere agradecer la financiación del proyecto Proyecto Prometeo II/2015/011, Generalitat Valenciana. El segundo autor agradece la financiación recibida por la beca predoctoral ACIF/2016/170, Generalitat Valenciana. Este trabajo ha sido financiado por el Proyecto de Innovación Docente PDI-DMA-2018 "Modelizaciones algebraicas de algunos juegos matemáticos", Departamento de Matemática Aplicada (UPV). Nos gustaría agradecer especialmente a Ana Martínez Pastor sus siempre valiosos comentarios.
} 


\begin{abstract}
Resumen
La asignatura Métodos Algebraicos y sus Aplicaciones del Máster en Investigación Matemática está orientada a mostrar la aplicabilidad que ofrecen algunas estructuras algebraicas abstractas en diversas áreas científicas, tanto en la propia matemática pura como en otras áreas más aplicadas de nuestro entorno real. Uno de los principales problemas en el aprendizaje de dichas estructuras es su alto grado de abstracción que dificulta su entendimiento, principalmente para alumnos que se inician por primera vez en este campo, y la falta de aplicabilidad con las que han sido tratadas con anterioridad, centrándose en su aspecto puramente matemático.

El método de utilizar juegos en la enseñanza es una innovadora y eficaz herramienta conocida como "game-based learning". Esta tendencia pedagógica es utilizada como componente lúdica con un objetivo concreto de aprendizaje. En esta línea, la presente comunicación trata sobre la modelización matemática de diversos puzzles y rompecabezas, así como el estudio de su resolubilidad, mediante técnicas algebraicas que han sido utilizadas en la citada asignatura para abordar el estudio de la estructura de grupo y el análisis de sus propiedades más básicas. Para ello, hacemos uso en el aula del sistema algebraico computacional GAP (Groups, Algorithms and Programming), el cual facilita los cálculos en álgebra discreta y posibilita una mejor comprensión de esta estructura. El caso del cubo de Rubik, uno de los juegos más célebres y vendidos en el mundo, y sus variantes son considerados especialmente.
\end{abstract}

Keywords: Álgebra computacional, teoría de grupos, modelización, puzzles, GAP.

\title{
1 Introducción
}

La asignatura Métodos Algebraicos y sus Aplicaciones forma parte del plan de estudios del Máster en Investigación Matemática, máster interuniversitario de la Universitat de València y la Universitat Politècnica de València. Este máster está orientado a iniciar a los alumnos en las técnicas básicas de la investigación matemática actual, tanto en el ámbito de la matemática pura como aplicada, formándolos en la adquisición de herramientas matemáticas de alto nivel y técnicas matemáticas avanzadas cubriendo las expectativas de graduados en matemáticas, ingenieros y otras ciencias básicas. La asignatura mencionada tiene un doble objetivo: profundizar en el estudio de las principales estructuras algebraicas tratadas de forma abstracta (semigrupos, monoides, grupos, anillos y cuerpos, álgebras, espacios vectoriales y módulos, retículos, anillos de polinomios, geometría algebraica, etc.) y en el conocimiento de una visión más aplicada de dichas estructuras en ámbitos tan diversos como la criptografía, teoría de códigos, teoría de autómatas y lenguajes formales, estructuras moleculares, teoría de grafos, resolución de ecuaciones diferenciales, etc. (véase Lidl y Pilz 1998).

Sin embargo, un hándicap importante a tener en cuenta en la impartición de esta asignatura es la gran diversidad del alumnado que la cursa, pues podemos encontrarnos titulados en: física, ingeniería, informática, arquitectura, matemáticas, etc. El desajuste de conocimientos matemáticos adquiridos y la diversidad en la formación previa con la que acceden estos estudiantes influyen sustancialmente en la forma 
de presentar las unidades didácticas y la forma de trabajar en el aula. Así podemos encontrar alumnos con una formación matemática amplia pero una visión aplicada insuficiente y, al mismo tiempo, alumnos con una previa formación matemática más deficitaria pero, sin embargo, con unas capacidades prácticas mucho más trabajadas. Una de las principales preocupaciones docentes es pues mitigar este desajuste, adaptando la programación de la asignatura al alumnado matriculado de forma que suscite el mayor interés y atención en todos los estudiantes, aumentando de esta forma su motivación en el aula y manteniendo, al mismo tiempo, las expectativas particulares de cada estudiante. Por otro lado, otro de los principales retos es dar una visión más aplicada de los conocimientos abstractos matemáticos cuya falta puede observarse en los alumnos con una formación más teórica.

En la presente comunicación mostramos algunas técnicas desarrolladas en la citada asignatura con el fin de paliar los problemas expuestos anteriormente, utilizando la modelización matemática de algunos juegos y puzzles matemáticos que son familiares a los estudiantes y analizando su posible resolución. En esta misma línea, muchos profesionales han optado por utilizar de forma exitosa diversas técnicas, alternativas a la usual clase presencial, como son la gamificación, los serious games o el gamebased learning. Estas técnicas presentan ciertas diferencias entre sí, pero todas ellas nos permiten utilizar el juego como herramienta auxiliar del aprendizaje. Diversos estudios ponen de manifiesto que los formatos gamificados de formación mejoran la retención y la asimilación de contenidos teóricos que pueden resultar bastante tediosos. El enfoque motivador de los juegos educacionales convierte el proceso de enseñanza y aprendizaje en algo dinámico e interesante, y ayuda al estudiante-jugador a expandir su conocimiento, consolidar y reforzar los conceptos ya aprendidos e investigar nuevas ideas que en ellos puedan surgir.

El caso del cubo de Rubik, uno de los rompecabezas más célebres en el mundo, y sus diferentes variantes, así como otros puzzles que pueden jugarse libremente de forma online, han sido especialmente tratados por tener un alto contenido algebraico implícito y por proporcionar ejemplos sencillos de grupos de simetrías cuyas propiedades más básicas pueden ser analizadas de forma exhaustiva por los estudiantes. Hemos realizado una modelización de dichas simetrías utilizando la teoría de grupos, y abordamos su resolución en el aula mediante el sistema algebraico computacional GAP de libre acceso (véase la referencia The GAP Group s.f.). De esta forma, tenemos la ventaja de utilizar la tecnología disponible a nuestro alcance, fomentando los conocimientos informáticos y el manejo de dispositivos digitales, imprescindibles en la actualidad para el desarrollo personal y profesional de nuestros titulados. Concretamente en nuestro caso, estos conocimientos resultan necesarios para iniciarse en la computación matemática.

\section{Objetivos}

La teoría de grupos es conocida tradicionalmente como la teoría matemática de la simetría. Ello es debido a que la estructura de grupo aparece de manera natural al considerar el conjunto de todas las aplicaciones biyectivas que mantienen una estructura determinada. El uso de la teoría de grupos no queda confinado solo al ámbito de la matemática pura, sino que la idea de grupo como medida de simetría desempeña un importante papel en otras ramas del saber científico, como es el caso de la química, 
la física, las ciencias de la computación y teoría de la información, la arquitectura e incluso en ciencias humanas y sociales.

La definición de la estructura de grupo es abstracta y en muchas ocasiones no resulta fácil de comprender para el alumnado. Sin embargo, los diferentes movimientos de ciertos puzzles y rompecabezas llevan implícitos el concepto de permutación y aparece de forma natural el grupo de las permutaciones permitidas. La idea es analizar propiedades básicas y profundizar en la estructura de los diferentes grupos de permutaciones que aparecen en el estudio de dichos movimientos. Desde un punto de vista docente, este tipo de técnicas pueden ayudar a comprender algunos conceptos teóricos de la teoría de grupos (como grupo libre, grupos de permutaciones, grupo simétrico y alternado, homomorfismos de grupos, construcciones de grupos como producto directo, producto semidirecto y producto orlado, etc.) y ver como aparecen de forma natural ejemplos sencillos sin necesidad de usar construcciones teóricas tediosas y mucho más artificiales.

En la presente comunicación presentamos la modelización de algunos rompecabezas matemáticos sencillos usando conceptos básicos de la teoría de grupos y analizado su resolubilidad. Sin embargo, los métodos de resolución de los mismos conllevan unos cálculos que resultan inviables de realizar sobre el papel. El software utilizado ha sido GAP (Groups, Algorithms and Programming) el cual es un programa especializado en la computación en teoría de grupos y álgebra discreta. Este software fue desarrollado en el Lehrstuhl D für Mathematik (LDFM), en RWTH Aachen, Alemania, desde 1986 hasta 1997. Después el desarrollo de GAP y su mantenimiento fue coordinado por School of Mathematical and Computational Sciences en la Universidad de St. Andrews, Escocia. En el verano de 2005 la coordinación fue de nuevo transferida a una asociación de 4 'Centros GAP', localizados en la Universidad de St. Andrews, RWTH Aachen, el Technische Universität Braunschweig y la Universidad Estatal de Colorado. La elección de dicho programa se ha debido principalmente a que es de libre distribución, lo cual es fundamental para que los alumnos puedan consolidar posteriormente en sus hogares los conceptos aprendidos en el aula, y su actualización es constante como puede comprobarse en The GAP Group s.f.

En resumen, nuestro principal objetivo en el aula es doble: por un lado, modelizar algunos puzzles y rompecabezas utilizando conceptos básicos de teoría de grupos y, por otro lado, ilustrar algunos métodos de resolución de los mismos mediante el uso del software GAP. Algunas de estas modelizaciones pueden encontrarse en un artículo docente que está siendo sometido en la actualidad (Felipe y Ortiz Sotomayor 2018). Con ello se pretende que los alumnos trabajen con aplicaciones reales de la teoría de grupos, mediante la formulación de problemas cotidianos que les son familiares, poniendo en valor el uso de la abstracción teórica y la modelización matemática. 


\section{Desarrollo de la innovación}

La asignatura Métodos Algebraicos y sus Aplicaciones del Máster en Investigación Matemática se imparte en el segundo cuatrimestre y tiene asignados 3 créditos ECTS. Normalmente utilizamos un par de sesiones, de dos horas cada una de ellas, en las que introducimos los conceptos teóricos necesarios e ilustramos la modelización matemática de rompecabezas tales como el cubo de Rubik y algunas variantes. Comenzamos modelizando el famoso cubo inventado por el escultor y profesor de arquitectura húngaro Erno Rubik, en 1974, y definimos el grupo de permutaciones Rubik asociado a sus movimientos básicos. Utilizando permutaciones y homomorfismos de grupos analizamos su resolubilidad en el caso de que sea posible, como sucede con los posibles "huevos fritos" que podemos formar en el cubo (ver Figura 1).

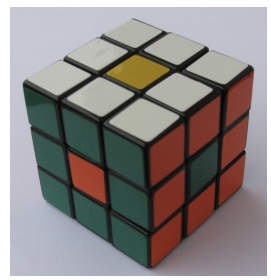

Fig. 1: Un cubo de "huevos fritos"

Posteriormente, entregamos diferentes cubos a los alumnos desordenados y se les insta a su formalización matemática (ver Figura 2). Los estudiantes trabajan in situ los conceptos impartidos en el aula en grupos de 2 o 3 personas, obteniendo su resolución mediante el programa GAP. Una vez resuelto este puzzle, se ensaya con otras modelizaciones análogas de rompecabezas que son variaciones del famoso cubo, como son el caso de los cubos 4x4, 5x5, el cubo Skewb y otras variantes geométricas como es el caso del Pyraminx que tiene una estructura piramidal (véase las Figuras 3, 4, 5 y 6 ).
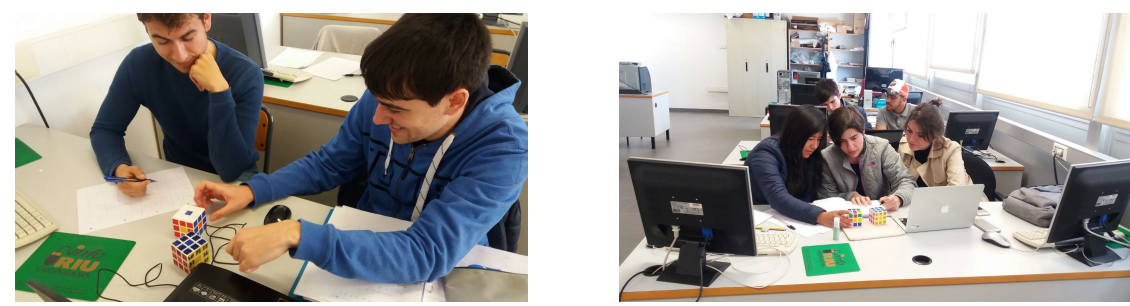

Fig. 2: Alumnos modelizando matemáticamente el cubo de Rubik

Consideramos también el caso de la resolubilidad de los famosos puzzles de deslizamientos (Figura 7) que nos permitirán motivar la introducción de la paricidad de las permutaciones y los grupos alternados. Posteriormente, se plantean algunos ejercicios con otros juegos que pueden modelizarse de una forma similar aplicando grupos de permutaciones como los anillos tricolores de Rubik (Figura 8) y el juego Top Spin, para que sean trabajados de forma autónoma en casa. Para ello, se les facilita a los alumnos la referencia Scherphuis s.f. donde se recopilan una gran cantidad de juegos de este tipo, que pueden jugarse libremente de forma online. La idea es que los estudiantes analicen e investiguen las nuevas estructuras que aparecen y se cuestionen 
sus diferentes variantes. Los ejercicios propuestos son expuestos en exposiciones orales frente al resto de sus compañeros para compartir los resultados obtenidos y para que sean debatidas las técnicas utilizadas.

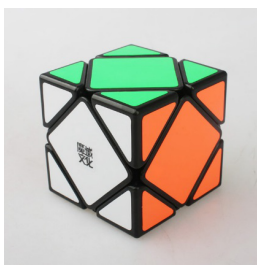

Fig. 3: Cubo Skewb

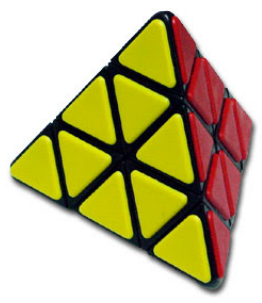

Fig. 5: Pyraminx

\begin{tabular}{||c|c||c||c||}
\hline 1 & 2 & 3 & 4 \\
\hline 5 & 6 & 7 & 8 \\
\hline 9 & 10 & 11 & 12 \\
\hline 13 & 15 & 14 & \\
\hline \hline
\end{tabular}

Fig. 7: Puzzle del deslizamiento $4 \times 4$

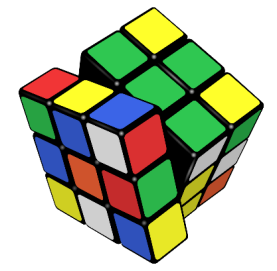

Fig. 4: Cubo de Rubik en movimiento

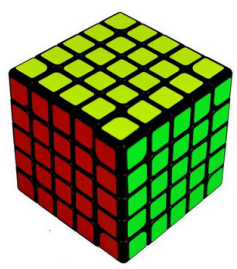

Fig. 6: Cubo de Rubik 5x5

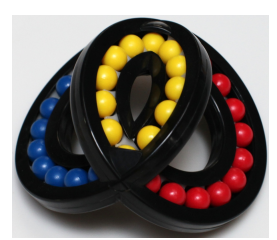

Fig. 8: Anillo tricolor de Rubik

Hemos de señalar que las experiencias que hemos tenido estos últimos años en clase son satisfactorias y que los alumnos muestran un buen ambiente de trabajo. Personalmente, debemos destacar un claro aumento de la motivación, la cual se desea continuar potenciando a lo largo de los próximos cursos académicos. Se ha pasado al final de curso una encuesta sobre la satisfacción de las actividades docentes que se han llevado a cabo, las cuales los alumnos han respondido de manera anónima, obteniendo datos objetivos de dichas actividades y pudiendo así planificar una lista de posibles mejoras, las cuales debatimos en la siguiente sección. Es importante decir también que las técnicas utilizadas en estas modelizaciones son una buena manera de que el alumnado desarrolle otras competencias transversales más específicas como son la resolución de problemas, aplicación y pensamiento práctico, trabajo en equipo y liderazgo y comunicación efectiva entre otras. Las actividades lúdicas programadas con las modelizaciones matemáticas de estos entretenimientos nos permitirán obtener nuevos parámetros para poder evaluar estas competencias, como por ejemplo la comunicación efectiva, de la que la citada asignatura es punto de control. Mencionar que ser punto de control de una competencia transversal implica plantear actividades para, en el desarrollo de los contenidos, trabajar la competencia transversal y evaluarla, recogiendo evidencias de los logros alcanzados. 


\section{Resultados}

Tal y como hemos comentado en la sección anterior, las experiencias previas de cursos anteriores en los que hemos ido introduciendo la innovación mencionada de forma inicial y las opiniones que nos han manifestado los alumnos implicados han sido muy satisfactorias. Sin embargo, en este curso académico hemos realizado unas encuestas, las cuales los alumnos han rellenado anónimamente, con el fin de valorar el impacto y obtener datos objetivos del proceso.

El número total de alumnos matriculados este año en la asignatura ha sido de 7 . Hemos realizado las siguientes 14 cuestiones, las cuales los alumnos han valorado eligiendo una de las opciones de respuesta: TED - Totalmente en desacuerdo, MBD - Más bien en desacuerdo, IND - Indeciso, MBA - Más bien de acuerdo, TDA - Totalmente de acuerdo. A continuación, resumimos en unos histogramas los resultados obtenidos:

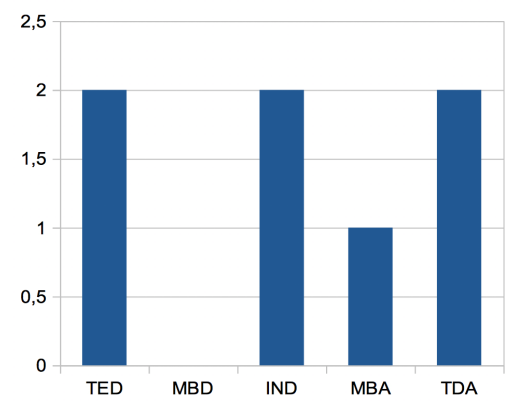

Fig. 9: ¿Crees que tu nivel de conocimiento teórico de las estructuras algebraicas era bueno antes del comienzo del curso?

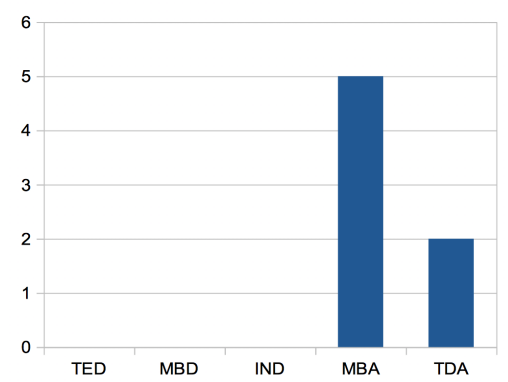

Fig. 11: ¿Consideras que los contenidos impartidos en la asignatura son importantes para complementar tu formación matemática?

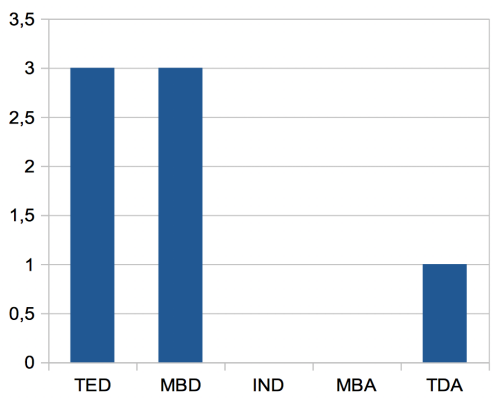

Fig. 10: ¿Crees que tu conocimiento sobre aplicaciones prácticas de las estructuras algebraicas era bueno antes del comienzo del curso?

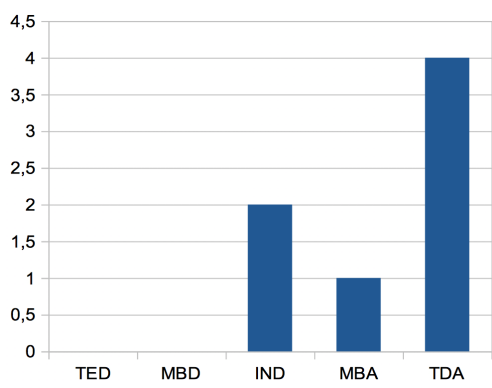

Fig. 12: ¿Consideras que la distribución de contenidos teórico-prácticos de la asignatura son adecuados?

A la vista de los resultados, podemos sacar varias conclusiones. Con la Figura 9 comprobamos cómo los conocimientos teóricos de las estructuras algebraicas que posee el alumnado al comienzo del curso es bastante diverso, tal y como comentamos en la introdución, debido principalmente a la diversidad de las titulaciones con las que acceden al máster. También vemos cómo en general escasea el conocimiento de aplicaciones prácticas de dichos conocimientos teóricos (Figura 10). Queda presente que los alumnos que cursan la asignatura finalizan bastante satisfechos con los contenidos 


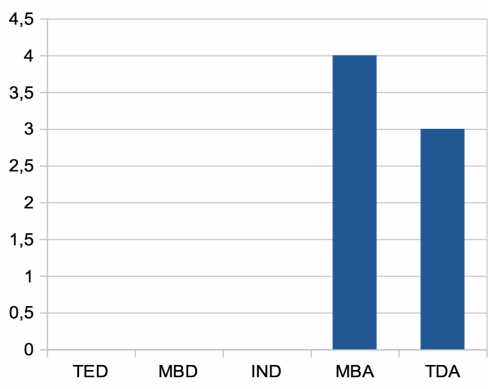

Fig. 13: ¿Crees que la asignatura te ha servido para aumentar dicho nivel del conocimiento teórico?

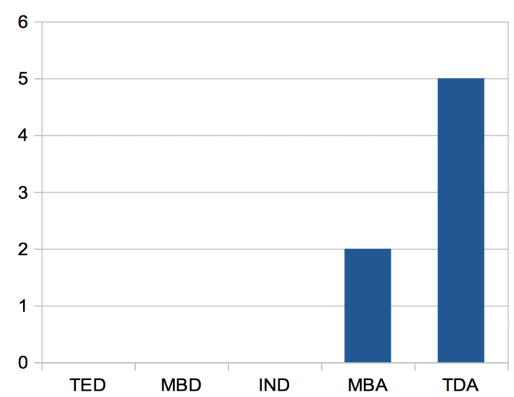

Fig. 14: ¿Crees que el curso te ha servido para aumentar dicho nivel de aplicaciones prácticas?

impartidos, tanto teóricos como prácticos, como se observa en los histogramas de las Figuras 11, 12, 13 y 14.

De hecho, cabe destacar la valoración en general positiva que realizan del software GAP y de las modelizaciones llevadas a cabo:

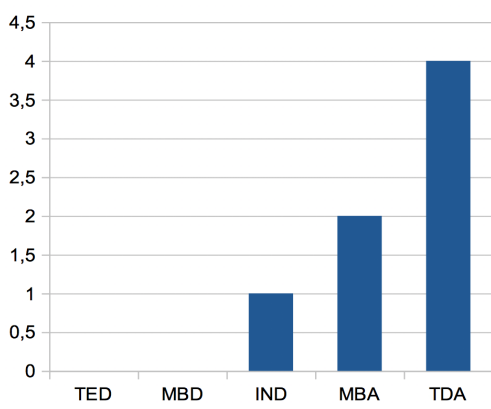

Fig. 15: ¿Consideras que el uso del software algebraico GAP te ha ayudado en la comprensión y manejo de las estructuras algebraicas?

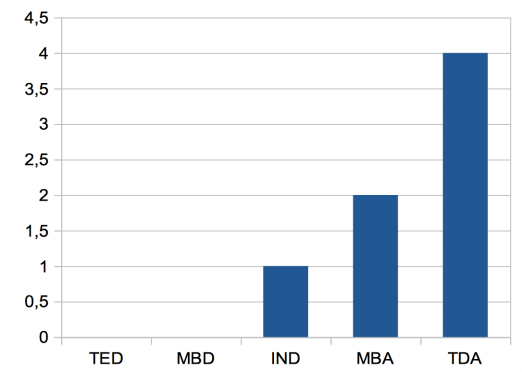

Fig. 17: ¿La práctica relativa a la modelización de puzzles (cubo Rubik) con GAP te resultó interesante?

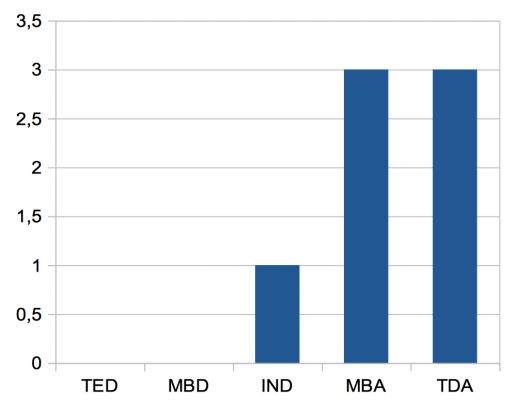

Fig. 16: En general, ¿te han resultado motivadoras las clases de GAP?

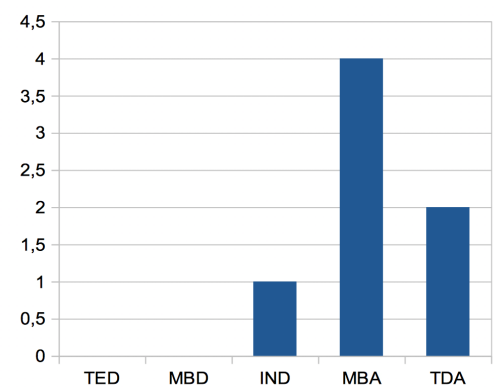

Fig. 18: ¿Crees que deberían plantearse más ejemplos de modelización que motiven los conceptos teóricos impartidos en la asignatura?

Las Figuras 15 y 16 muestran cómo el software GAP ayuda al alumnado a la comprensión de las estructuras algebraicas y aumenta la motivación de los mismos, los 


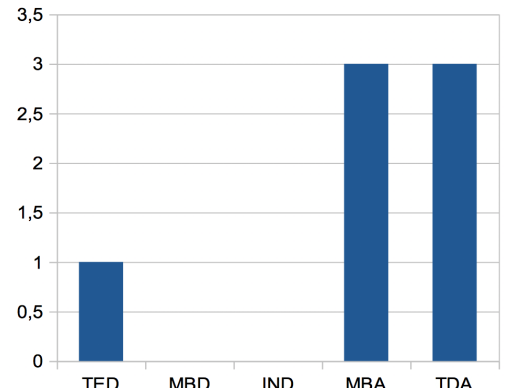

Fig. 19: ¿Crees conveniente conocer modelizaciones que hagan uso de programas algebraicos (como GAP, Singular, Magma, etc?) para abordar aplicaciones a problemas reales?

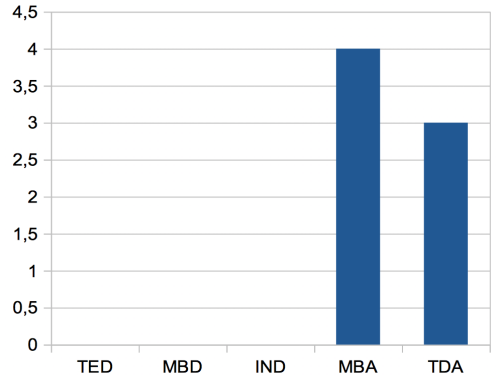

Fig. 20: En general, ¿consideras que los contenidos impartidos cubren las expectativas que inicialmente tenías de la asignatura?

cuales eran dos objetivos principales a conseguir. Con las Figuras 17, 18 y 19 vemos el agrado mayoritario de los alumnos con modelizaciones que motiven los conceptos teóricos explicados en clase y con el uso de programas algebraicos que ayuden en los cálculos. Con la última figura (20) queda de manifiesto la satisfacción general de los estudiantes con los contenidos impartidos a lo largo de la asignatura.

Finalmente, queremos señalar también unos últimos apartados de las encuestas dedicados a la libre opinión sobre la asignatura, de donde destacamos las siguientes manifestaciones de los alumnos:

"Aunque los contenidos hacia final de curso se han ido complicando, los conceptos han quedado claro gracias a los ejemplos."

"Creo que la asignatura se ha adaptado bastante bien a los diferentes niveles de los alumnos."

"Personalmente busco ejemplos aplicados, y al presentar al final de la materia una aplicación del interés del estudiante nos permite investigar y ampliar más nuestros conocimientos en el campo que queramos formarnos profesionalmente o académicamente."

\section{Conclusiones}

Podríamos pensar que los rompecabezas y puzzles pueden servir únicamente de entretenimiento y diversión. Sin embargo, dichos entretenimientos pueden entenderse como juegos realmente educativos, no solo porque desarrollan nuestro intelecto, nuestra visión espacial, capacidad lógica y de razonamiento, sino porque constituyen además una eficaz herramienta en el entendimiento de algunas estructuras algebraicas que, debido a su alto grado abstracción matemática, son de difícil comprensión. El estudio de los movimientos "legales" en cierto rompecabezas y las simetrías asociadas a estos movimientos permiten estimular el aprendizaje de las nociones más básicas de la teoría de grupos mediante permutaciones. En Joyner 2008 puede encontrarse una recopilación más amplia de otros juegos que pueden ser tratados de forma similar a 
los presentados en esta comunicación. Lo más relevante es que hemos apostado por un tratamiento más computacional de dichas modelizaciones, tanto respecto a su estudio como a la resolución de estos puzzles, mediante la utilización del sistema algebraico GAP. De esta forma se pretende utilizar la tecnología disponible a nuestro alcance, a la cual están acostumbrados nuestros estudiantes, para fomentar las habilidades informáticas imprescindibles en la actualidad para el desarrollo de la matemática computacional, necesaria tanto a nivel docente como investigador.

De forma general, con las actividades y técnicas presentadas en la presente comunicación en la modelización de juegos se pretende principalmente ayudar al aprendizaje de las estructuras algebraicas de una forma lúdica, conseguir que los alumnos se "enganchen" por la materia independientemente de su abstracción matemática. También desarrollar habilidades y destrezas asociadas a ciertas competencias transversales tales como la resolución de problemas, pensamiento crítico y comunicación efectiva, así como incentivar su interés por la materia y disfrutar de los contenidos aprendidos mejorando al mismo tiempo el compromiso de los estudiantes en su propio aprendizaje.

A la vista de todos los datos, en resumen podemos calificar la innovación docente realizada como muy satisfactoria. Para finalizar, destacar que en la actualidad continuamos trabajando con otras modelizaciones matemáticas que involucran estructuras algebraicas alternativas, como son los anillos de polinomios haciendo énfasis en las bases de Gröbner, las cuales poseen multitud de aplicaciones como es el caso de las coloraciones de grafos, teoría de códigos, resolución de curvas algebraicas, ecuaciones diferenciales y, en relación con los entretenimientos matemáticos, la resolución de sudokus. Para estas nuevas modelizaciones estamos utilizando los programas GAP y Singular, que es un sistema de álgebra computacional de manipulación polinomial con especial énfasis en álgebra conmutativa, geometría algebráica y teoría de la singularidad y el cual es también de libre distribución.

\section{Referencias bibliográficas}

Felipe, M.J. y V.M. Ortiz Sotomayor (2018). "Jugando con la Teoría de Grupos: rompecabezas, puzzles y otros entretenimientos matemáticos". En: sometido.

Joyner, D. (2008). Adventures in group theory: Rubik's cube, Merlin's machine, and other mathematical toys. Baltimore: The Johns Hopkins University Press.

Lidl, R. y G. Pilz (1998). Applied Abstract Algebra. New York: Springer-Verlag.

Scherphuis, J. Jaap's puzzles page. URL: https://www.jaapsch.net/puzzles.

The GAP Group. GAP - Groups, Algorithms, and Programming. Ver. 4.8.10. URL: http://www.gap-system.org. 